\title{
Virtual prototyping used as validation tool in automotive design
}

\author{
$\underline{\text { A Kulkarni }}^{\text {a }}$, A Kapoor ${ }^{\text {a }}$, M Iyer ${ }^{\text {b }}$ and V Kosse ${ }^{\text {b }}$ \\ ${ }^{a}$ Faculty of engineering and Industrial Science, Swinburne university of Technology, \\ John Street, Hawthorn, VIC-3122, Australia \\ ${ }^{b}$ Faculty of Built environment and engineering, Queensland University of Technology, \\ Brisbane, QLD 4001 Australia. \\ Email: ambarishkulkarni@swin.edu.au
}

\begin{abstract}
Virtual prototyping (VP) is more commonly used alternative to rapid prototyping (RP) to validate products without substantial investments. This article covers recent advancements in VP technologies to accommodate kinematics and use them to evaluate functional aspects of automotive designs. Traditionally to validate effective function of the product, prototypes were constructed in the earlier stages of design. These prototypes were used to validate design functions of new products. Subsequently products were launched for production runs after corrections were made for issues found during prototyping stages. VP techniques use advancements in software tools to mimic the physical prototyping of product. The new advancements of using function parameters in soft tools added new paradigm in VP techniques. Kinematics with function parameters such as load conditions, motions are added using force (F) and motion (M) functions in design scenario. Kinematics used in virtual environment uses positions, velocity, acceleration, inertial forces, and power requirements, of all the components in mechanisms to recreate virtual effect on the models. Comprehensive engineering designs are produced reducing product design cycle by saving time, costs in set ups and manufacturing the physical prototypes. This extension of virtual design process for physical sampling is unique and was developed, implemented successfully in automotive design application. In this paper experimental study conducted on a car carrier, which is developed under authors responsibility has been used to illustrate VP techniques.
\end{abstract}

Keywords: Virtual prototyping, rapid prototype, Kinematics, automotive, car carrier 


\section{INTRODUCTION}

End of twentieth century marked use of CAE techniques, which enhanced capabilities in engineers for reducing design cycle times of product and costly prototypes. Simulation technology was used which was accessible and usable by design engineers, people on the front line who made most of decisions. However simulation was limited to fluid flow and traditional CAE, until the use of VP techniques which took into next level of design engineering. In general, product market is becoming customer driven causing shorter market life cycles. This customer focused market increases pressure for cost reduction in over all products. Traditional way of designing and rapid prototyping has to be reevaluated to incorporate new technologies to reduce any possible cycle time and beyond. This reduction in cycle time is achieved by reducing design cycle time and time needed to generate prototypes, which uses most of time in overall design cycles.

Under fierce development globally, approach of incorporating VP in product development to validate products is becoming common practice. Up till now RP had an upper hand over VP with physical performance however with introduction of dynamic evaluations, VP can be used effectively to perform quality design validation. In this research technological advancement in field of simulation was used to new level of kinematics and dynamic analysis before product is launched. The rigid body techniques were used in conjunction with virtual environment to simulate physical prototyping, which validated different parameters during car carrier development. The VP approach is an extension of virtual design process, consisting of development of two dimensional schematics initially and then developing a virtual reality model. RP approach had a longer cycle time due to prototype build time and correction times for tooling and other associated fixtures. Technological advancements have led digital verifications of products using VP techniques. Another advantage in using VP techniques was to conceptualise and communicate in multidisciplinary teams (Wang, Shen et al. 2002). Design undergoes several numbers of design cycles and variations in design reviews until a final design is settled. Generally physical prototyping is used to validate the effectiveness of design function. The VP techniques reduce the costs and life cycle by visualising and determining problems at a very early stage of the design process. This visualisation is integrated in an interactive 3D user interface that enables rapid design decisions and modifications when compared to physical prototyping. The effectiveness of VP is demonstrated in this paper using car carrier design.

Product design industry is increasingly hampered to reduce the design life cycle in order to cut costs and increase quality standards. Costs, technology and productivity are important for survival of automotive industries. In product life cycle, product development stage is crucial, involving concept generations, and integration of concepts with functional requirements (Friedlaender, Winston et al. 1983). The basic components of product design cycle are design, drafting and validation. The design, drafting are electronically generated and validation is both electronic and physical building of a product. The development cycle is time consuming process, mainly due to conceptual and prototyping stages. These two activities are time consuming and play a vital role in product development cycle. In order to reduce design cycle time, evaluation of these two stages is essential. VP is a new technique used to reduce prototype construction time, since there is no physical construction of prototype. Virtual reality is obtained at no additional time expense, because everything needed to perform motion simulation has been defined in CAD assembly model already. In VP basic models are transferred to motion simulation program. These processes not only avoid using physical prototyping techniques, but also validate products in all stages of designs. Thus in today's sustainable environment use of virtual prototyping is becoming popular with virtual enterprising designs in automotive industries (Camarinha-Matos, Afsarmanesh et al. 1998). Product marketability is increased with use of VP techniques allied with concurrent engineering. Effectively called as collaborative virtual engineering, reduces product development cycles and enhances design quality (Chen and Liang 2000). The VP technique using motion simulation also checks for interferences, and this is a very different process from interference checking available with CAD assembly animation. Motion simulation conducts interference checks in real time, and provides the exact spatial and time positions of all mechanism components as well as the exact interfering volumes. This process not only solves analytical problems but also can be extended to generate the new parts in the assemblies using trajectories of motion into CAD geometry. For example based on the trajectories, track mechanism for car seven tracks in car carrier was developed. The motion data can be exported to FEA automatically or manually which makes it more interactive and subsequently used as a master model concept. This common, integrated environment covers all three tools including CAD, FEA and motions for data exchange facilities. This integration avoids data loss via neutral file formats, typical to standalone applications. In addition, use of motion simulation integrated with $\mathrm{CAD}$, greatly reduces efforts required to set up motion simulation models. With all these advantages, it also identifies issues in design and communicates interactively in a real-time without prototyping (Lau, Mak et al. 2003). This facilitates and streamlines effective development of modern automotive product. Use of these techniques over physical prototyping is explained in rest of the paper. 


\section{VIRTUAL PROTOTYPING}

The product development of a car carrier was complex process, but rather a sequence of decisions and adjustments until it was completely developed. Therefore, high risk was involved in development stemmed from making too many decisions without knowing if they were the best ones. If problems were discovered late in the design process, it will be tough time recovering quickly or inexpensively. The risk in development was minimised using VP techniques, which periodically confirmed that design was headed on right directions. Different prototypes were prepared and verified to formulate the final car carrier designs (Guidea S. and Nye T. J. 2005). Though VP techniques are essential, RP techniques have upper hand over them due to functional assessment of the products (Liang and Wei 2006; Venables M. 2006). Prototype vehicle is essential to validate the product performance (Consano L. and Colombano M. 2007). In the second half of the last century virtual reality (VR) was used mainly to verify assembly of designs (Jayaram, Connacher et al. 1997; Gomes De SA and Zachmann 1999; Jayaram, Jayaram et al. 1999) and some researchers used it with integration of collaborative engineering techniques (Dahan and Srinivasan 2000; Shyamsundar and Gadh 2001; Shyamsundar and Gadh 2002). This collaborative environment achieves sharing of design data and effective use of man power across the globe (Tseng, Jiao et al. 1998; D'Adderio 2001). The maintenance activities like disassembling functions also used VP techniques to illustrate functions (Siddique and Rosen 1997; Srinivasan, Figueroa et al. 1999). Some advancements of VP were used for line balancing techniques for virtual assembly planning and virtual ergonomic prototyping applications (Bullinger, Richter et al. 2000). The VP techniques enhance determining complexity in products and simplify them. This is achieved by use of system enhanced interaction tools and modalities ( $\mathrm{Li}$ and Chai 2002). Creating virtual design is explained by Foley (Don and Foley 1997 ) and Myer (Mayer R.E. 2002), using the 3D max studio software. In our research, Solid works animator and Solid works modeling techniques are utilised to generate the virtual prototyping.

\subsection{Math generation}

Initially math models are built for different parts using CAD software. Design starts with vector based sketcher, which then are used to develop the features to complete the math model. Parametrised sketches are converted to block type files (.blk) and animations are created using sketcher. These sketcher animations used in car carrier design resulted in refining ideas, generating concepts and facilitating problem solving. The styled surface or the feature based techniques were also used in combination with sketcher for developing model to required dimensions. Hybrid modeling approach is generally used in automotive industry consisting of both parametric and non-parametric ways of constructing models. Use of design automation tools for construction of models like weldments, gear wizards, user defined tools, enhanced design cycle time reductions in car carrier design. Workflow management tools and groupware were also used, so as to communicate and share documents, images, 2D, 3D models, and 3D animations or simulations, hence proposed product was examined by all the design team during stages of development. As described in the book 'Animation tips and tracks for windows and Mac' Foley (Don and Foley 1997 ), the TARGA (.tga) files was used to set up the virtual environment required. Chunky solid methods use boolean operations, till required shape is achieved. The models for cars and different concept parts including decks, frame and structures were created in this stage for car carrier.

\subsection{Functional scenario}

One of key issue in car carrier research was driver's falling from heights above 1.5 meters ground level. Generally drivers load cars and strap cars on top deck which is usually more than recommended 1.5 meters. Research on design was intended to identify areas of 1.5 meters above ground level and design a system which can resolve issue of potential falls. Hence VP techniques were used during concept development stages to evaluate loading, unloading sequence of car carriers, to validate concepts and functional aspects of identified solutions. Virtual prototypes were developed and design cases were presented to illustrate the feasibility. All the car decks concepts were created in two stages. At initial stage sketch block methods were used to demonstrate the feasibility of the concept. This avoided hours of work required to create models

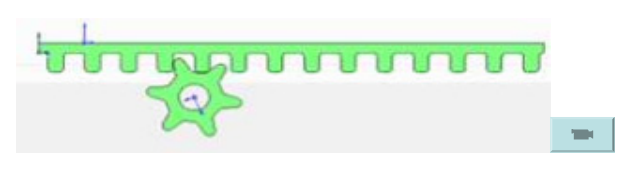

Figure 1. Rack and pinion simulation

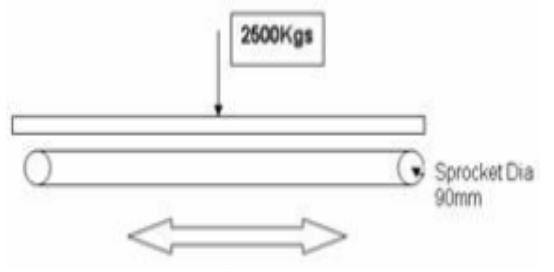

Figure 2. Schematic model of deck mechanism 
or rapid prototypes to demonstrate the concepts. This avoided hours of work required to create models or rapid prototypes to demonstrate the concepts. Reevaluation is done at every stage of design when models were created. The functional aspects of designs were parametrised using the kinematics to study motion behaviors of system elements. In addition virtual environments were added to mimic real functioning of hydraulics and motors. These assessments refined designs to fine tune for exact functions of the system elements. One of functional assessment derived from car carrier was rack and pinion mechanisms. These assessments refined designs to fine tune for exact functions of the system elements. One of functional assessment derived from car carrier was rack and pinion mechanisms. It was evaluated using VP techniques (figure 1) which converts motions from rotary and linear. The rack is flat, toothed part, the pinion is gear which converts from rotary to linear (GonzÂjilez-Palacios and Angeles 2003). The diameter of the gear determines the speed with which rack moves as the pinion turns. Based on the distance traveled by deck, numbers of revolutions are calculated in gear profile using equations 1 to 5 as shown below. When work is done in a specified time, power is used.

WORK $($ Ft. Pounds $)=\mathrm{W} \times 2 \pi \mathrm{R} / 12 \times$ No. of Rev. of shaft.

POWER (Ft. Pounds per Min.) $=\mathrm{W} \times \mathrm{RPM}$

$\mathrm{HP}=\mathrm{W} \times 2 \pi \mathrm{R} / 12 \times \mathrm{RPM} / 33,000$

TORQUE (Inch Pounds) $=$ FORCE $(\mathrm{W}) \times$ RADIUS $(\mathrm{R})$

HORSEPOWER $(H P)=$ TORQUE $(T) \times$ RPM $/ 63500$

When selecting the right gear, horsepower, torque and duty cycle (operating conditions) were three of functional parameters considered. In addition, there were two other important variables center distance and ratio in order to meet speed (rpm) requirements. Five variables listed above horsepower, torque, duty cycle, center distance and ratios, pitch of pinion -rack (assuming the center distance and ratio are fixed) were fed into the functional parameter field for kinematics analysis (equations 6 and 7):

$\mathrm{PD}$ of pinion $=2 \times$ center distance $/$ ratio +1

Pitch of Rack $=$ PD of pinion $\times$ ratio

To ascertain the basic theoretical displacements, torque requirement of the motor was calculated. Schematic models used for calculations deck designs are used to in to virtual models to evaluate function parameters (figure 2). Data: Diameter of the sprocket $\mathrm{D}=$ $90 \mathrm{~mm}$, Load on the deck F'= $2500 \mathrm{Kgs} 2500 \mathrm{KgF}$. Transfer speed/ time $\mathrm{V}=$ Apx 3.6 meters $/ 20 \mathrm{sec}$ ie 11.4 meters $/ \mathrm{min}$, Considerations: Friction Factor $\mu=0.3, \pi=3.142, \mathrm{~g}=9.81 \mathrm{~N} \mathrm{~V}=\pi \mathrm{DN} / 1000$ and $11.4=3.142 \times 90 \times \mathrm{N} / 1000$

Where $\mathrm{V}=$ speed, $\mathrm{D}=$ Diameter and $\mathrm{N}=\mathrm{RPM}(\mathrm{N}=40$ revolutions $/ \mathrm{min}) \quad \operatorname{Load} \mathrm{F}=\mu \mathrm{F}^{\prime} \quad=0.3 \times 2500=750 \mathrm{Kgs}$

Total two motors, $375 \mathrm{~kg}$ 's each side $=\mathrm{F}=375 \times 9.81=3680 \mathrm{~N} \mathrm{~m}$, Torque $\mathrm{T}=\mathrm{F} \times \mathrm{d}$

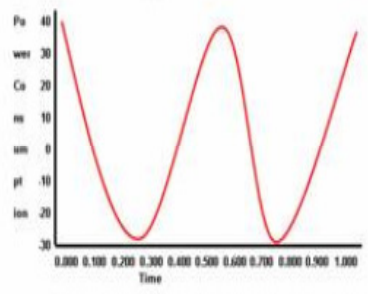

Figure 3. Motor Power consumption at time intervals

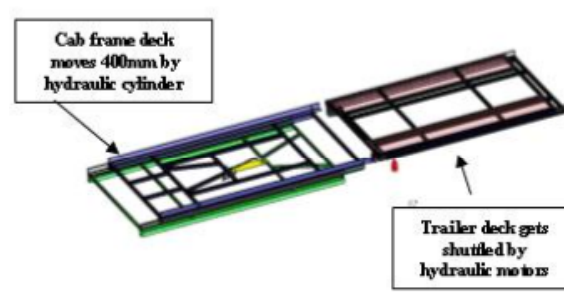

Figure 4.Virtual prototype for evaluating functional performance

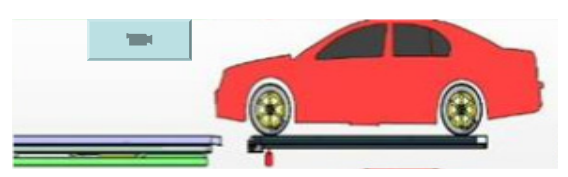

Figure 5.Virtual prototype for test frame

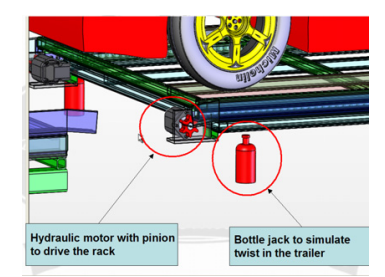

Figure 6.Virtual prototype simulation for test frame

$\mathrm{d}=$ Radius of sprocket and Torque $=3680 / \mathrm{N} \times 45 \mathrm{~mm}=165600 \mathrm{~N} \mathrm{~mm}=165 \mathrm{NM}$, Low speed motor of RPM 40-60 and torque 165NM was proposed, The higher torque motor MGR 100 was selected from the Rexroth, motor supplier tables Torque $\mathrm{T}=9550 \times$ Power $(\mathrm{KW}) / \mathrm{RPM}$ drive sprocket $\mathrm{P}=\mathrm{T} \times \omega$ $\omega$ Omega $=2 \pi \mathrm{N}$ (RPM-drive sprocket) $/ 60=4.2 \mathrm{rad} / \mathrm{sec}=0.69 \mathrm{KW}$

The motor selected would produce a torque of $165 \mathrm{NM}$ and low speed of 40 revolutions per minute. Once assemblies were completed with respective mates, speed of the motor, points to be traced, and motion results were fed as function parameters (equations 8 to 12) in virtual design study. The motion simulation program uses material properties from the CAD parts to define inertial properties of mechanism components, and translates CAD assembly mating conditions into kinematics joints. It then automatically formulates equations that describe the mechanism motion defined in initial studies. A numerical solver solves equations of motion very quickly, and results include full information about displacements, velocities, accelerations, joint reactions, and inertial loads of all mechanism components, as well as the power necessary to sustain required motion. The motor power consumption is calculated in figure 3 illustrate the simplicity of generating motion studies with the function parameters in VP. 


\subsection{Virtual Simulations}

Once the motion functions for speeds of motors and hydraulics are defined, virtual environment is created to add the back ground to models. The frame and all car carrier bodies are mated, placed with cars on decks. Functional parts like motor, hydraulics and chain drives are also used in evaluation for system integration analysis. Generally car carrier designs in VP are made as sub-assemblies to simplify handling. Such an example is rack and pinion used on deck car one mechanism prototype. In car carrier design, due to uncertainties and unexpected problems, virtual prototype was used as part of validation process to evaluate different design proposals, and confirm functionality before starting production of car carriers. Virtual prototyping explored process of cross-functional integration in new car carrier design (Nihtila 1996). The solids are geometrically constrained to control smooth motions in $3 \mathrm{D}$ environment where they were interpolated in a specific defined path (Rossignac J. R. and Kim J. J. 2001). The virtual camera was used to capture and create .avi with lighting effects. Once the camera is proposed, time line is generated about a given motion. The time line can be adjusted or edited in any stage to obtain desired start and end points at time intervals. Virtual prototype constructed as shown in figure 4 which is used for functional evaluation of deck prototype. The cab frame deck constructed consisted of hydraulic cylinder capable of pushing the track inside by desired $400 \mathrm{~mm}$. The deck one in frame is cassette type arrangement lifted with hydraulics. Once deck positions in front of cab deck, car is transferred across using rack and pinion mechanisms powered by motors simulated for functional performance (figure 5 and figure 6). Two screw jacks are mounted on front of the deck one to simulate the flex in deck of car carrier frame. The lateral forces are applied on frame body to bring it to real flexing in decks caused due to overhanging. Animation sequence is run on whole car carrier deck models, resulting in solver solving equations of motion to verify function parameters for displacements, velocities, accelerations, joint reactions, and inertial loads of all the mechanism components, as well as the power necessary to sustain the motion. Motion simulation conducted interference checks in real time, and provided the exact spatial and time positions of all mechanism components as well as the exact interfering volumes in car carrier. VP also validated the accommodations of different cars in regard to space and automation. The layout also validated the required regulatory requirements including ADR (Australian Design rule 43-04 1989) and transportation guides(1996). VP reduced design cycle, enhanced quality of design, space constrains, shapes and overall skeleton of car carrier design.

\section{RAPID PROTOTYPING}

In car carrier design, series of prototypes were designed, constructed and tested as final design emerged to prepare it for production. The purpose of prototypes was to progressively refine car carrier design. One of strategy used in car carrier design was to test, evaluate and modify design based on analysis of prototypes. The goal of the industry funding bodies including Tolls was to realise a prototype vehicle. Prototypes were built for car seven and car one decks to evaluate functional performance. The analyses of these two concepts were crucial, as operational automation was used to reduce risks of falls from heights. Rack and pinions were made to validate the designs by laser cutting the prototype designs. Car one and car seven frames were built as prototype test rigs and functional evaluations were carried (figure 7 and figure 8). The test rig was actuated by use of power pack and bottle jacks were placed on the front ends to reproduce some flex in decks as happens in actual car carriers. The motors were actuated on either ends provided enough torques to move the deck with load of car around 2.2 tons to the other deck. The motor and rack-pinion performances are studied during these cycles. The load thrust and the capability of rack and pinion to move it across validated working of whole system in integration with hydraulic

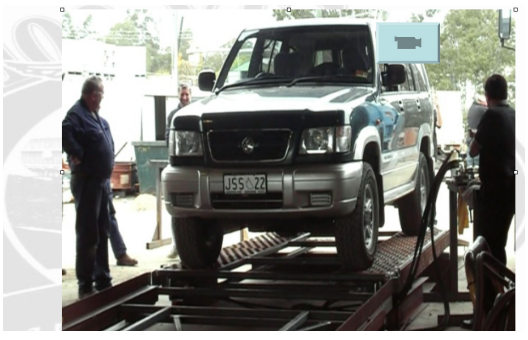

Figure 7.Rapid prototype for evaluating functional performance of deck one

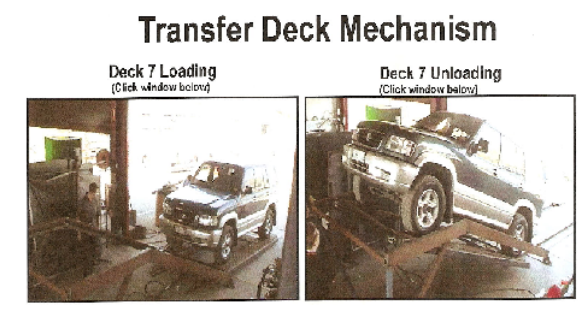

Figure 8.Virtual prototype for evaluating functional performance of deck seven

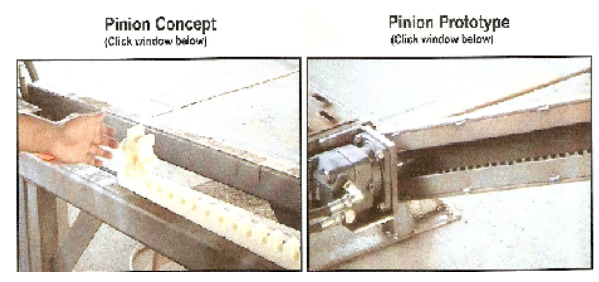

Figure 9. Rapid prototype of rack-pinions cylinders. It was important step in realizing functional aspect of whole deck car one systems to resolve historic problem of falls. RP techniques validated working principles of designed concepts, mechanisms and 
performances of car one and seven decks. During investigation using prototype test rigs car one deck prototype validated usability of rack and pinion mechanism. It was identified by using car seven deck prototypes that pinion failure was caused due to amplification of loads by gravity and its own weight. The gears and profiles started yielding due to these loads on long trial runs. These finding could identify advantages of RP techniques over VP in making product first time right. Other areas focused during rapid prototyping stage were use of several materials for gear design and different designed profile shapes to evaluate wear and tear caused during operational sequences (figure 9).

\section{DISCUSSIONS \& SUMMARY}

Virtual prototyping methodology is an extension of virtual reality. This techniques developed further has potential to replace traditional approach of rapid prototyping and test rigs (physical sampling methods). In this research extensive use of VP has resulted in minimal building of test rigs to validate car carrier concepts. The physical samplings of parts are time consuming as parts needed to be designed and then manufactured. Since design evaluation is closed loop system, defects found in rapid prototypes are then corrected in designs. If reuse of old test prototype is impossible, then new prototype construction is required to reevaluate the corrected model. This makes RP process not only time consuming, but also costly process, contributing substantial to market price of a product. For these reasons virtual models enhance qualitative validations at substantially small costs. The previous methods of using rapid prototyping (RP) are compared with VP techniques in this paper. Previously prototypes were built and engineering changes were evident throughout design evolution. The VP used lesser time compared to RP techniques and hence was economical. The earlier days without function parameters it was impossible to compare with RP techniques. Since RP technique was required for validation of operation parameter of product. The VP

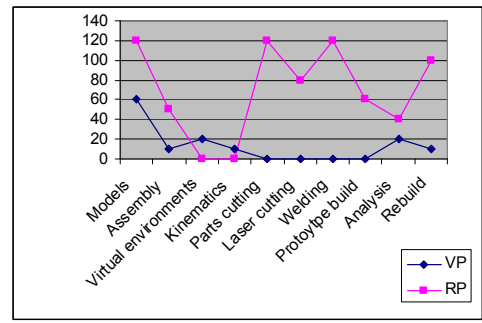

Figure 10.Comparisons of VP and RP systems

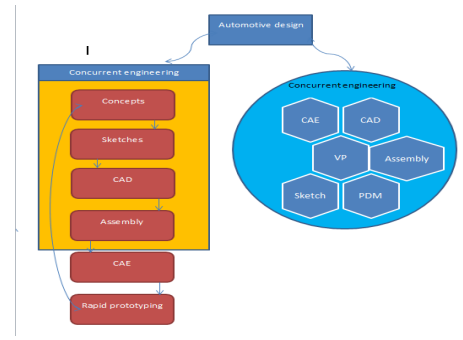

Figure 11.VP and RP systems techniques now are advanced and equipped with function parameters for defining kinematics. As shown in fig. 10, VP technique saved substantial time reducing overall market time of car carrier. Figure 11 demonstrates VP and RP techniques in collaborative environment and real time interaction with downstream applications. VP has upper hand in downstream applications including use of FEA and CAD packages makes this process suitable solution for a global sustainable agenda.

Recent industry developments have shifted their focus from structural performance to kinematics and dynamics of new products before building of physical prototypes. The mounting pressure to reduce product costs and faster time cycles have led to cutting down the product life cycle. Product validation, a part of development process is achieved by physical sampling using rapid prototyping method. This process validates effective functional performance of the product before it is launched for production. This process has two disadvantages mainly time consuming, as result it is expensive. Virtual prototyping is alternative method used to reduce development cycle time and subsequent costs involved. The results of virtual prototype are obtained virtually at no additional time expense, as parts used in VP techniques are modelled in the CAD already. In addition to mechanism analysis, product developers can also use motion simulation for mechanism synthesis by converting trajectories of motion into CAD geometry, and using it to create new part geometry. Motion simulation results supply of input data required for structural analysis conducted with FEA. Thus VP techniques have gained larger momentum with inclusion of design functions to effectively validate products kinematics and dynamics. These techniques embrace collaborative environment, sustainability concepts which are additional benefits. This leads to conclusion that a VP technique needs to be researched further for adoption as validation tools alternative to RP techniques in automotive designs.

\section{ACKNOWLEDGMENTS}

Author acknowledges the support of JSS and Tolls during development of novel car carrier. Author also thanks for assistance from Work safe and Queensland transport department for providing with requested data. 


\section{REFERENCES}

(1996). Heavy Vehicle Operators Guide. Brisbane, Queensland, Transport department,.

Australian Design rule 43-04 (1989). Vehicle Configuration and dimensions. Motor vehicle standard Act.

Bullinger, H. J., M. Richter, et al. (2000). "Virtual assembly planning." Human Factors and Ergonomics In Manufacturing 10(3): 331-341.

Camarinha-Matos, L. M., H. Afsarmanesh, et al. (1998). "Towards an architecture for virtual enterprises." Journal of Intelligent Manufacturing 9(2): 189-199.

Chen, Y. M. and M. W. Liang (2000). "Design and implementation of a collaborative engineering information system for allied concurrent engineering." International Journal of Computer Integrated Manufacturing 13(1): 11-30.

Consano L. and Colombano M. (2007). An optimized transport concept for tractor-semitrailer combination. VDI Berichte: $65-78$.

D'Adderio, L. (2001). "Crafting the virtual prototype: How firms integrate knowledge and capabilities across organisational boundaries." Research Policy 30(9): 1409-1424.

Dahan, E. and V. Srinivasan (2000). "Predictive power of Internet-based product concept testing using visual depiction and animation." Journal of Product Innovation Management 17(2): 99-109.

Don, M. and M. Foley (1997 ). Animation tips and tricks for windows And Mac, Peach pit press.

Friedlaender, A. F., C. Winston, et al. (1983). "COSTS, TECHNOLOGY, AND PRODUCTIVITY IN THE U. S. AUTOMOBILE INDUSTRY." Bell Journal of Economics 14(1): 1-19.

Gomes De SA, A. and G. Zachmann (1999). "Virtual reality as a tool for verification of assembly and maintenance processes." Computers and Graphics (Pergamon) 23(3): 389-403.

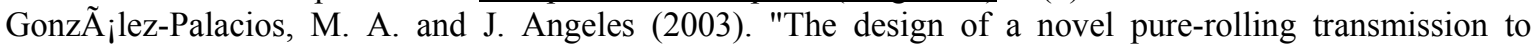
convert rotational into translational motion." Journal of Mechanical Design, Transactions of the ASME 125(1): 205-207.

Guidea S. and Nye T. J. (2005). Automated optimal design for manufacturability of sheet/plate assemblies. American Society of Mechanical Engineers, Design Engineering Division (Publication) DE, Orlando, FL.

Hsu, W. and I. M. Y. Woon (1998). "Current research in the conceptual design of mechanical products." Computer-Aided Design 30(5): 377-389.

Jayaram, S., H. I. Connacher, et al. (1997). "Virtual assembly using virtual reality techniques." $\underline{\text { CAD }}$ Computer Aided Design 29(8): 575-584.

Jayaram, S., U. Jayaram, et al. (1999). "VADE: A Virtual Assembly Design Environment." IEEE Computer Graphics and Applications 19(6): 44-50.

Lau, H. Y. K., K. L. Mak, et al. (2003). "A virtual design platform for interactive product design and visualization." Journal of Materials Processing Technology 139(1-3 SPEC): 402-407.

Li, B. H. and X. D. Chai (2002). "Virtual prototyping engineering for complex product." Jisuanji Jicheng Zhizao Xitong/Computer Integrated Manufacturing Systems, CIMS 8(9): 678-683.

Liang, J. S. and P. W. Wei (2006). "Conceptual design system in a Web-based virtual interactive environment for product development." International Journal of Advanced Manufacturing Technology 30(11-12): 1010-1020.

Mayer R.E. (2002). Multimedia Learning, Cambridge University Press.

Nihtila, J. (1996). "Integration mechanism in new product development." Acta Polytechnica Scandinavica Mathematics and Computing Series 78.

Rossignac J. R. and Kim J. J. (2001). "Computing and visualizing pose-interpolating 3D motions." Computer-Aided Design 33(4): 279-291.

Shyamsundar, N. and R. Gadh (2001). "Internet-based collaborative product design with assembly features and virtual design spaces." CAD Computer Aided Design 33(9): 637-651.

Shyamsundar, N. and R. Gadh (2002). "Collaborative virtual prototyping of product assemblies over the Internet." CAD Computer Aided Design 34(10): 755-768.

Siddique, Z. and D. W. Rosen (1997). "A virtual prototyping approach to product disassembly reasoning." CAD Computer Aided Design 29(12): 847-860.

Srinivasan, H., R. Figueroa, et al. (1999). "Selective disassembly for virtual prototyping as applied to demanufacturing." Robotics and Computer-Integrated Manufacturing 15(3): 231-245.

Tseng, M. M., J. Jiao, et al. (1998). "Virtual prototyping for customized product development." Integrated Manufacturing Systems 9(6): 334-343.

Venables M. (2006). "Better by design." Manufacturing Engineer 85(3): 24-27.

Wang, L., W. Shen, et al. (2002). "Collaborative conceptual design-State of the art and future trends." Computer Aided design 34. 